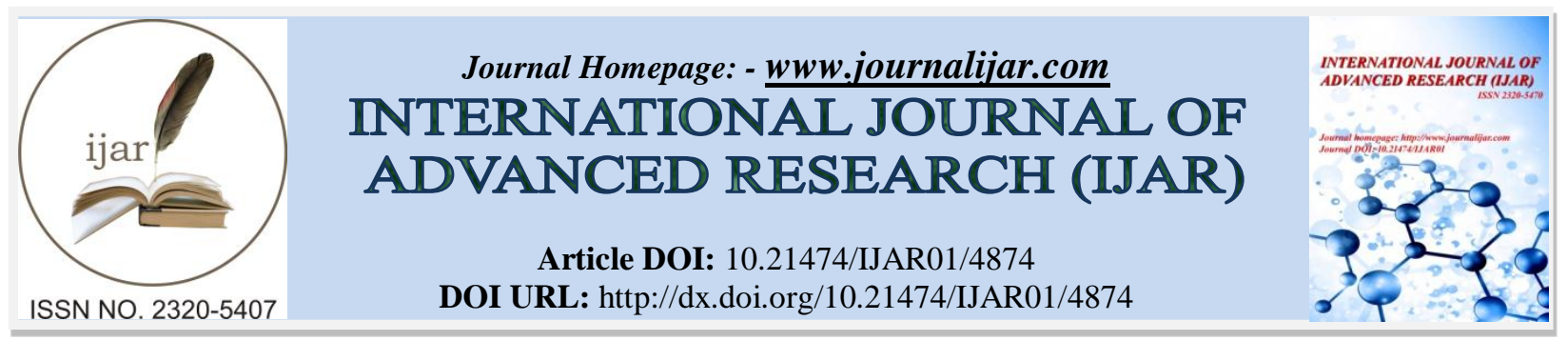

RESEARCH ARTICLE

\title{
PHYTOSYNTHESIS VS. TRADITIONAL CHEMICAL SYNTHESIS FOR OBTAINING BIOCOMPATIBLE DRUG DELIVERY (DDS) NANOPARTICLES: A REVIEW.
}

\section{"Gomaa F. Salma ${ }^{1}$ and El-Sherbiny M. Ibrahium ${ }^{2}$.}

1. Chemistry department, School of Science and Engineering, American University in Cairo, Cairo, Egypt.

2. Material Science department, Nanotechnology center, Zewail City for Science and Technology, Cairo, Egypt.

\section{Manuscript Info}

(.........................

Manuscript History

Received: 17 May 2017

Final Accepted: 19 June 2017

Published: July 2017

Key words:-

Green Chemistry, nanoparticles, drug

delivery, mono-dispersed.

\section{Abstract}

Recently, the applications of nanoparticles (NPs) in nanomedicine have gained a lot of interest. They can be used for biological labeling, biosensors and as therapeutic agents. As a result, there is an urgent need to develop synthesize techniques that can result in more biocompatible Nps, suitable for biomedical applications . Chemical methods for the preparation of NPs are simple, easy to perform and very variable. However, their main disadvantages is the use of toxic chemicals [2, 3]. In the search of better pathway for synthesizing metal Nps that can overcome these limitations, researchers have turned to biological systems. Biosynthetic (biogenic) techniques employ proteins, microbes or plant extract for nanoparticles production. The target is to implement safer and biocompatible synthetic methods that eliminate using harmful chemical reagents, thus has no/less harmful impact on human beings. Phytosynthesis of NPs using plant extracts have been extensively investigated. Plant extract contains intrinsic phytochemicals such as saponins, terpenoids, proteins, polyphenols and flavonoids, having the properties of stabilizers/ emulsifiers. It can be used for the synthesis of biocompatible, monodisperesd NPs of minimum particle size. In comparison to other biological synthesis techniques, Nps synthesized using plants are more stable and the synthesis rates are more rapid. Problems associated with complex treatments (e.g. microbial isolation, culturing, maintenance) are resolved in the case of phytosynthesis. Furthermore, the controlled flexibility in the size and morphology of obtained NPs are considered higher in case of phytosynthesis due to the diverse candidates of plants. This review provides an overview of recent trends in the phytosynthesis of NPs, expected mechanism for the biosynthesis process, and their potential advantage in the field of drug delivery.

Copy Right, IJAR, 2017,. All rights reserved.

\section{Introduction:-}

Metal nanoparticles (Nps) are widely used in number of applications, including electronics, catalysis, and sensors technology. Recently, the applications of metal NPs in nanomedicine have gained a lot of interest. They can be used for biological labeling, biosensors and as therapeutic agents. As a result, there is an urgent need to develop synthesize techniques that can result in more biocompatible metal Nps, suitable for biomedical applications [1]. 
Generally speaking, there are two main approaches for synthesizing metal Nps, physical methods and chemical methods. Physical methods of synthesize is a typical "top-down" approach. It starts with a bulk material which is reduced in size by means of mechanical methods such as attrition and laser ablation. The advantage of this technique is the narrow size distribution, while its limitation is the need for expensive instruments, such as laser. The second direction is the chemical methods, particularly wet chemical methods. It is considered "bottom-top" approach in which metal ions are reduced under certain conditions, forming small aggregates of metal atoms. Chemical methods are simple, easy to perform and very variable. However, their main disadvantages is the use of toxic chemicals [2, 3]. In the search of better pathway for synthesizing metal Nps that can overcome these limitations, researchers have turned to biological systems. Biosynthetic (biogenic) techniques employ proteins, microbes or plant extract for nanoparticles production. Such green approach provides advantages over traditional chemical and physical methods since it is non-toxic, fast, and environment friendly. This review provides a brief overview for the biosynthesis of metal nanoparticles, gold and silver in particular, using plant extract. This is followed by discussion for the expected mechanism for the biosynthesis process. Finally, current applications of biosynthesized metal Nps in drug delivery are presented [1,4].

General principles for the synthesis of solution-based colloidal nanoparticles:-

Solution-based colloidal method is the most common technique used for synthesizing nanoparticles. This process involves two main steps, nucleation followed by subsequent growth. Nucleation takes place when the concentration of solute reaches a certain value above the solubility constant. At this point, nucleation is initiated and the concentration, or supersaturation, decreases gradually with time, until it reaches a value below the nucleation threshold. At this point, no further nucleation takes place, and subsequent growth dominates. Subsequent growth proceeds by incorporating growth species, available in the solution, into the existing nuclei. This process continues until the concentration of the growth species reaches the solubility constant i.e. no more available growth species to be incorporated in the particles (Fig 1.a). Both nucleation and subsequent growth needs to be controlled in such way to obtain smaller size NPs with uniform size distribution i.e. monodisperse NPs. For the synthesis of small NPs with uniform size distribution, the nucleation process must occur very rapidly, in a short period of time. In this case, all nuclei are formed at the same time, with almost the same size (since they are formed at the same conditions). Furthermore, subsequent growth of these nuclei must be appropriately controlled $[2,5,6]$.

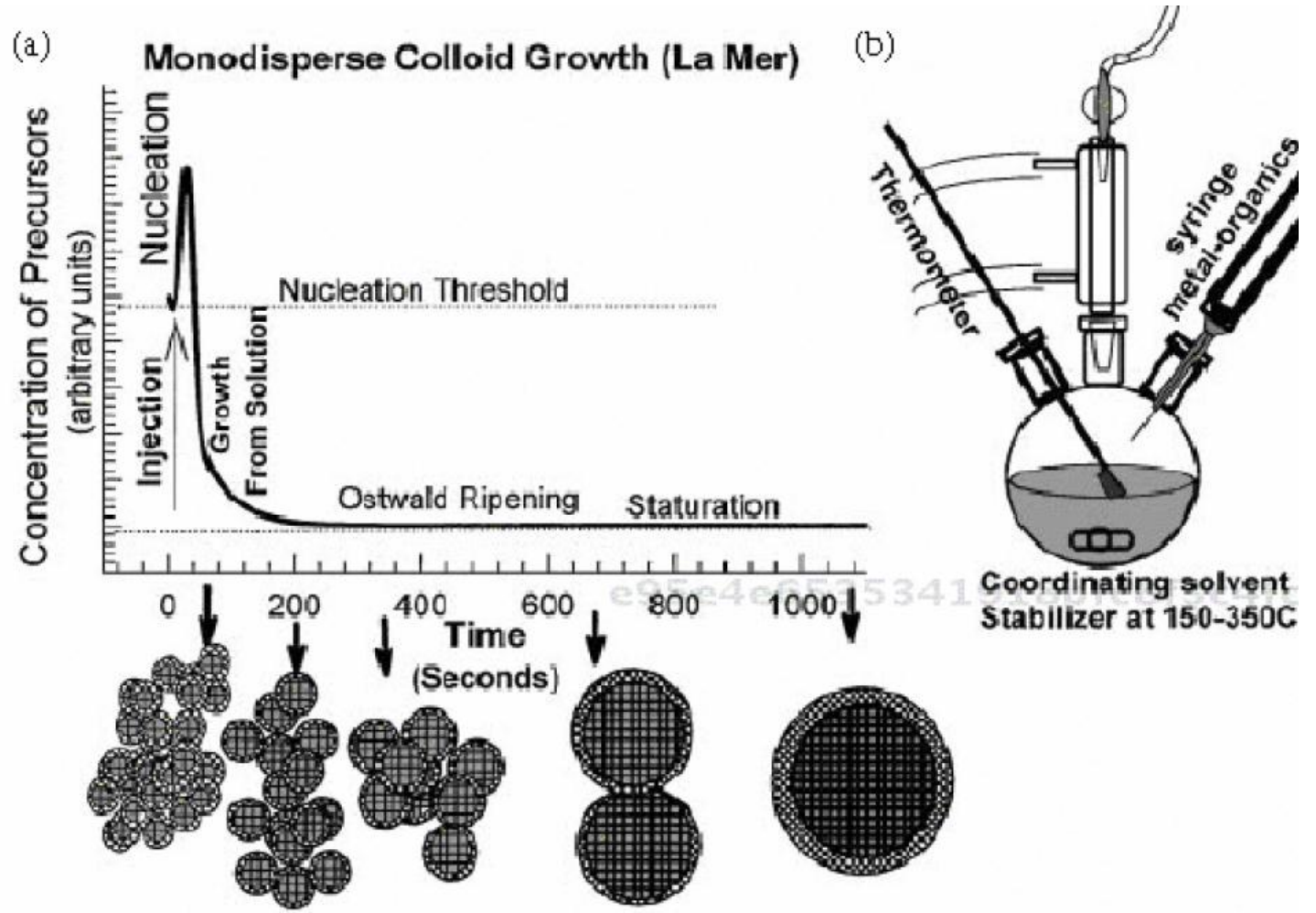

Fig 1:- a) Schematic presentation for the stages of nucleation and growth for the preparation of monodisperse NPs,

b) Representation of the simple synthetic apparatus employed in the preparation of monodisperse NPs [5]. 
Rapid nucleation: Practically, sharp nucleation can be achieved by the rapid addition of reactants to the reactor. This will increase the concentration of the growth species abruptly to a very high supersaturation. Consequently, large numbers of nuclei are formed in a short period of time. Generally, for a given concentration, large number of nuclei means smaller size nuclei $[2,6]$

Controlling subsequent growth: size and size distribution of NPs depend on the subsequent growth of the existing nuclei. In general, the growth process consists of two main steps. First is the diffusion step. This step includes diffusion of growth species from the surrounding solution, followed by adsorption on the growth surface. Second is the growth step which includes the incorporation of the already adsorbed species into the surface, resulting in a surface growth $[2,5,6]$.

For the synthesis of monosized NPs, limiting the diffusion step, which includes limiting diffusion and adsorption of growth species, is highly desired. In other words, diffusion-limited process can result in a monodisperse NPs. There are several ways to achieve diffusion-limited growth. For example, when the concentration of growth species is kept extremely low, diffusion distance would be very large and diffusion becomes the limiting step. This can be achieved by working at high dilution of solute with momentaneous supersaturation (rapid addition of reagents). Such momnetaneous superstaturation can be achieved by rapid addition of reagent. This would allow for nucleation to occur in a short period of time. Furthermore, increasing the viscosity of solution can acts as a diffusion barrier. Addition of surfactant molecules is a possible approach to obtain diffusion-limited growth. Surfactant molecules act as a capping material. They are adsorbed on the surface of particle, rending it unavailable for further growth $[2,5,6]$

Many systems exhibit a second growth phase called Ostwald ripening. In this process, smaller particles, which have high surface energy, dissolve while larger nanoparticles grow. As a result, the numbers of nanoparticles decrease overtime, while the average particle size increases. Ostwald ripening can be exploited in the preparation of nanoparticles with different sizes. However, if monosized nanoparticles are required, the colloidal suspensions must be extracted from the reaction mixture to avoid Ostwald ripening. This can be achieved by using a syringe, applied during the reaction or reflux process (Fig 1.b) [2].

\section{Synthesis of metal nanoparticles:-}

The general method used for the synthesizing metal nanoparticles, or more specifically, metallic colloidal suspension, is the reduction of metal complexes (metal salts), with an appropriate reducing agent. The synthesis of metal colloidal suspension involves nucleation and subsequent growth. In the growth stage, presence of capping material plays an important role in determining the size and shape of nanoparticles. If the material is two weakly associated with the metal Nps, there will be a limited or no protection, thus, the formed NPs continue to grow into large size particles and finally aggregate. On the other hand, if the material is strongly bounded to the particle surface, the growth can be limited or even stopped. Consequently, it is possible to control the size and shape of metal nanoparticles by selecting the appropriate capping agent [2,3]. The main approach used to achieve monosized metallic nanoparticles is a combination of using a low concentration of solute and a polymeric compound as a capping agent. Both a low solute concentration and a polymeric surfactant would hinder the diffusion of the growth species from the surrounding solution to the growth surface. As a result, the diffusion process becomes the rate limiting step of the subsequent growth of initial nuclei, resulting in the formation of monosized nanoparticles. Various types of metal complexes (precursors), reduction reagents, and polymeric stabilizers were used to promote and control the reduction reactions, the initial nucleation and the subsequent growth of initial nuclei. Table 1 summarizes the precursors (metal complexes), reduction reagents, and polymeric stabilizers commonly used in the production of metallic colloidal dispersions $[2,3,7]$.

A wide range of metal nanoparticles have been synthesized, including copper, platinum, Cadmium, Iron, selenium....etc. Silver and gold nanoparticles are the commonly synthesized metal nanoparticles since they have a wide range of applications different aspects. For gold nanoparticles, the reduction of chlorauric acid at $100{ }^{\circ} \mathrm{C}$ in the presence of PVA as a stabilizer is the most commonly used method [2,7] 
Table1:- Summary of precursors, reduction reagents and polymer stabilizers [2].

Precursors Formula

Metal anode

$\mathrm{Pd}, \mathrm{Ni}, \mathrm{Co}$

Palladium chloride

$\mathrm{PdCl}_{2}$

Hydrogen hexachloroplatinate IV

$\mathrm{H}_{2} \mathrm{PtCl}_{6}$

Potassium tetrachloroplatinate II

$\mathrm{K}_{2} \mathrm{PtCl}_{4}$

Silver nitrate

$\mathrm{AgNO}_{3}$

Silver tetraoxylchlorate

$\mathrm{AgClO}_{4}$

Chloroauric acid

$\mathrm{HAuCl}_{4}$

Rhodium chloride

$\mathrm{RhCl}_{3}$

Reduction reagents

Hydrogen

Sodium citrate

Hydroxylamine hydrochloride

Citric acid

Carbon monoxide

Phosphorus in ether

Methanol

Hydrogen peroxide

Sodium carbonate

Sodium hydroxide

Formaldehyde

Sodium tetrahydroborate

Ammonium ions
$\mathrm{H}_{2}$

$\mathrm{Na}_{3} \mathrm{C}_{6} \mathrm{H}_{5} \mathrm{O}_{7}$

$\mathrm{NH}_{4} \mathrm{OH}+\mathrm{HCl}$

$\mathrm{C}_{6} \mathrm{H}_{8} \mathrm{O}_{7}$

$\mathrm{CO}$

$\mathrm{P}$

$\mathrm{CH}_{3} \mathrm{OH}$

$\mathrm{H}_{2} \mathrm{O}_{2}$

$\mathrm{Na}_{2} \mathrm{CO}_{3}$

$\mathrm{NaOH}$

$\mathrm{HCHO}$

$\mathrm{NaBH}_{4}$

$\mathrm{NH}_{4}^{-}$

Polymer stabilizers

Poly(vinylpyrrolidone), PVP

Polyvinylalcohol, PVA

Polyethyleneimine

Sodium polyphosphate

Sodium polyacrylate

Tetraalkylammonium halogenides 
Similarly, Ag nanoparticles are synthesized by UV illumination of aqueous solutions containing $\mathrm{AgClO}_{4}$, acetone, 2propanol and polyethyleneimine polymer as a stabilizer. Such photochemical reduction results in Ag nanoparticles about $7 \mathrm{~nm}$ in size with a narrow size distribution [7].

\section{Plants used in biosynthesis of metal nanoparticles (Green approach):-}

Biosynthesis of nanoparticles is a wet chemical technique. It is similar to traditional chemical methods in the fact that reduction is the main reaction for the generation of growth species. An important branch of metal Nps biosynthesis is the application of plant extract to the synthesis reaction. The antioxidant properties of plant phytochemicals are responsible for the reduction of metal compounds into their respective nanoparticles. The rate of synthesis of nanoparticles by plant extracts is comparable to those of chemical methods and faster than green synthesis by microorganisms. Furthermore, problems associated with complex treatments in case of microbial synthesis, including microbial isolation, culturing, and maintenance are resolved in the case of phytosynthesis. Another advantage for the phytosynthesis is the controlled flexibility in terms of size and morphology due to the diverse types of plants that can be used $[1,8,9]$.

Preparation of AgNPs and AuNps was first reported using Chrysopogon zizanioides. C. zizanioides, commonly known as vetiver, is a perennial grass commercially used to extract fragrance oils and has several medicinal properties. An aqueous leaf extract was added to silver nitrate $\left(\mathrm{AgNO}_{3}\right)$ and chloroauric acid $\left(\mathrm{HAuCl}_{4}\right)$ to synthesis silver and gold nanoparticles respectively. In case of AgNPs, the color of the reaction mixture changed from yellow to brown within 30 minutes of adding the leaf extract. Such color change indicated for the bioreduction of $\mathrm{Ag}^{+}$into $\mathrm{AgNps}$ (Fig 2.a and b). On the other hand, addition of leaf extract to $\mathrm{HAuCl}_{4}$ results in a ruddy red color within $2 \mathrm{hr}$, indicating for the bioreduction of $\mathrm{Au}^{+3}$ into AuNPs (Fig 2.c and d). The color change noticed for both AgNPs and AuNPs is attributed to the phenomenon of surface Plasmon resonance (SRP) which takes place in metals [4]. Furthermore, synthesize of AgNPs and AuNPs was characterized with ultraviolet-visible spectroscopy (UV-VIS). The appearance of peak is due to SRP, and it is characteristic for each metal NPs. AgNPs showed an absorption peak at $420 \mathrm{~nm}$, which is characteristic for silver nanoparticles (Fig 3). Similarly, the reaction mixture of AuNPs showed a characteristic peak for AuNPs at $540 \mathrm{~nm}$ (Fig 4) [4].

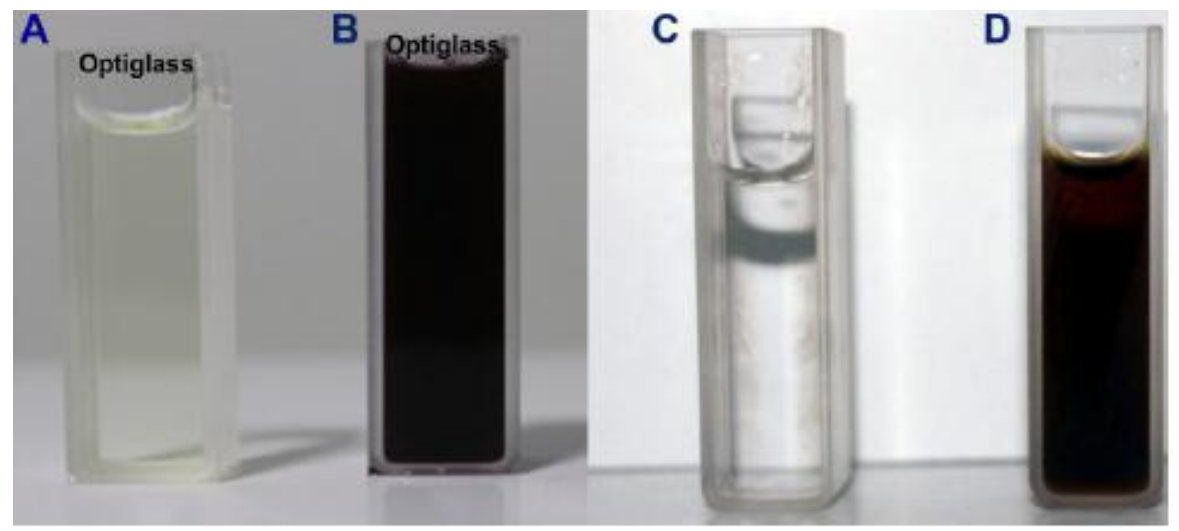

Fig 2:- Synthesis of silver and gold nanoparticles. (a) $\mathrm{AgNO}_{3}$; (b) synthesized AgNPs (brown color) solution after 24 hours; (c) $\mathrm{HAuCl}_{4}$ solution; (d) synthesized AuNPs (ruby red color) after 24 hours [4]. 


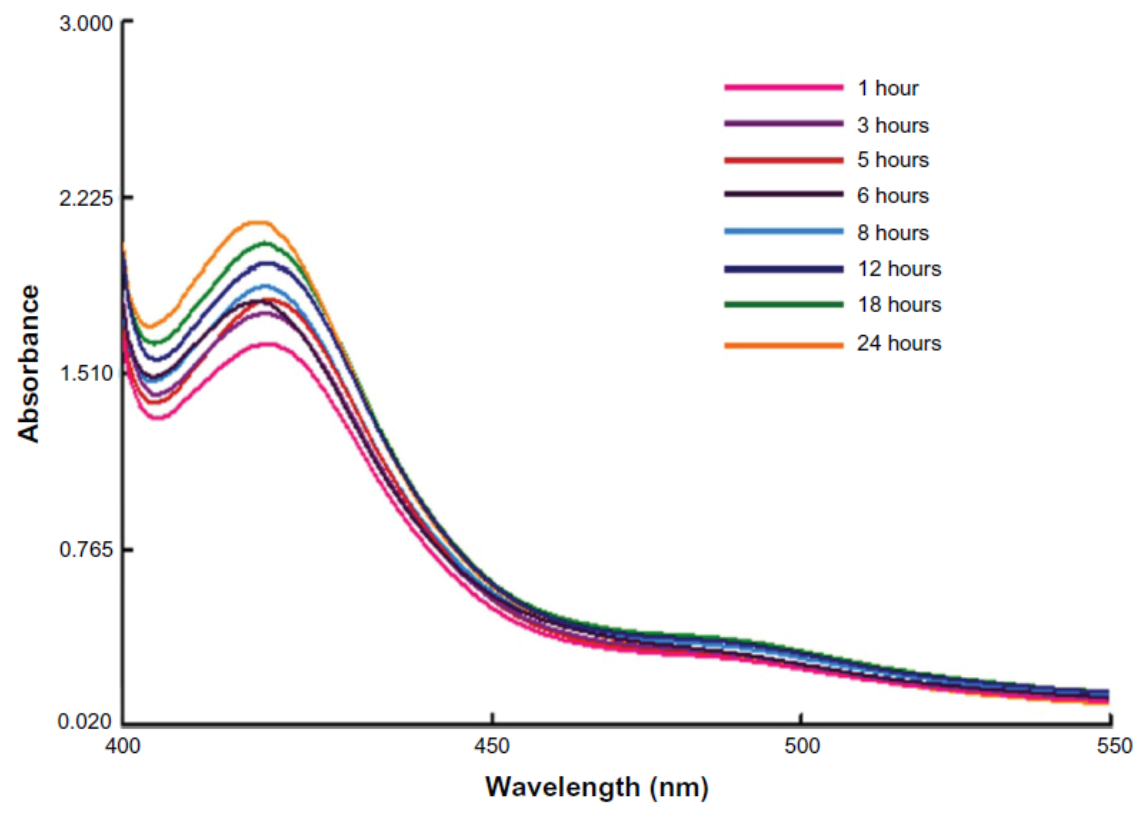

Fig 3:- Time dependent absorption spectra of silver nanoparticles after the bioreduction of silver in the aqueous extract of Chrysopogon zizanioides[4]

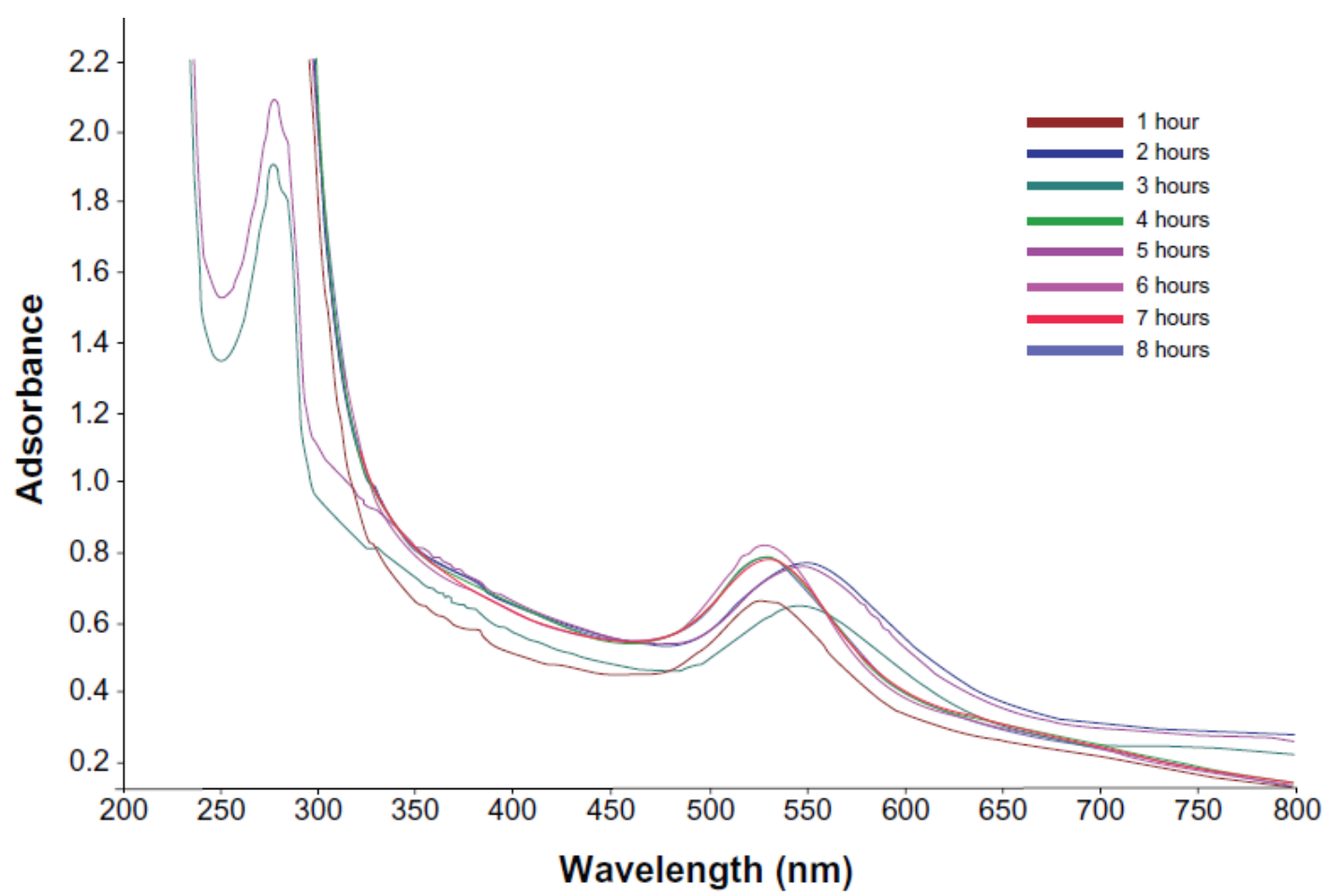

Fig 4:- Time dependent absorption spectra of gold nanoparticles after the bioreduction with aqueous extract of Chrysopogon zizanioides [4].

\section{Mechanism of nanoparticles synthesis of plant:-}

A vast range of secondary metabolites is found in all plants. They are involved in the vital processes occurring in plant cells. Phytochemical analysis of several plant extracts shows the presence of biomolecules such as carbohydrates and proteins, and other metabolites, such as flavonoids, steroids, and terpenoids. Such phytochemicals posses' redox capacity which makes them act as reducing agents. In addition, other phytochemical can act as stabilizers (capping agents) during nanoparticles synthesis $[9,10]$. 
In 2007, Li et al. proposed a recognition-reduction-limited nucleation and growth model which explains the production of metal NPs via plant extract (Fig 5). In the first stage, metal ions are trapped on the surface of metabolites present in plant extract through electrostatic interactions. In the second stage, metal ions are reduced by the action of these metabolites. In the third stage, nuclei grew by continuous reduction of metal ions and accumulation on these nuclei. In the fourth stage, reducing metabolites, and large number of biomolecules which act as stabilizers, lead to an isotropic growth of NPs, forming spherical NPs. Finally, with an increase in aging time, large-sized silver nanoparticles are formed and the crystalline phase changed from polycrystalline to single crystalline through Ostwald ripening $[9,11,12]$.

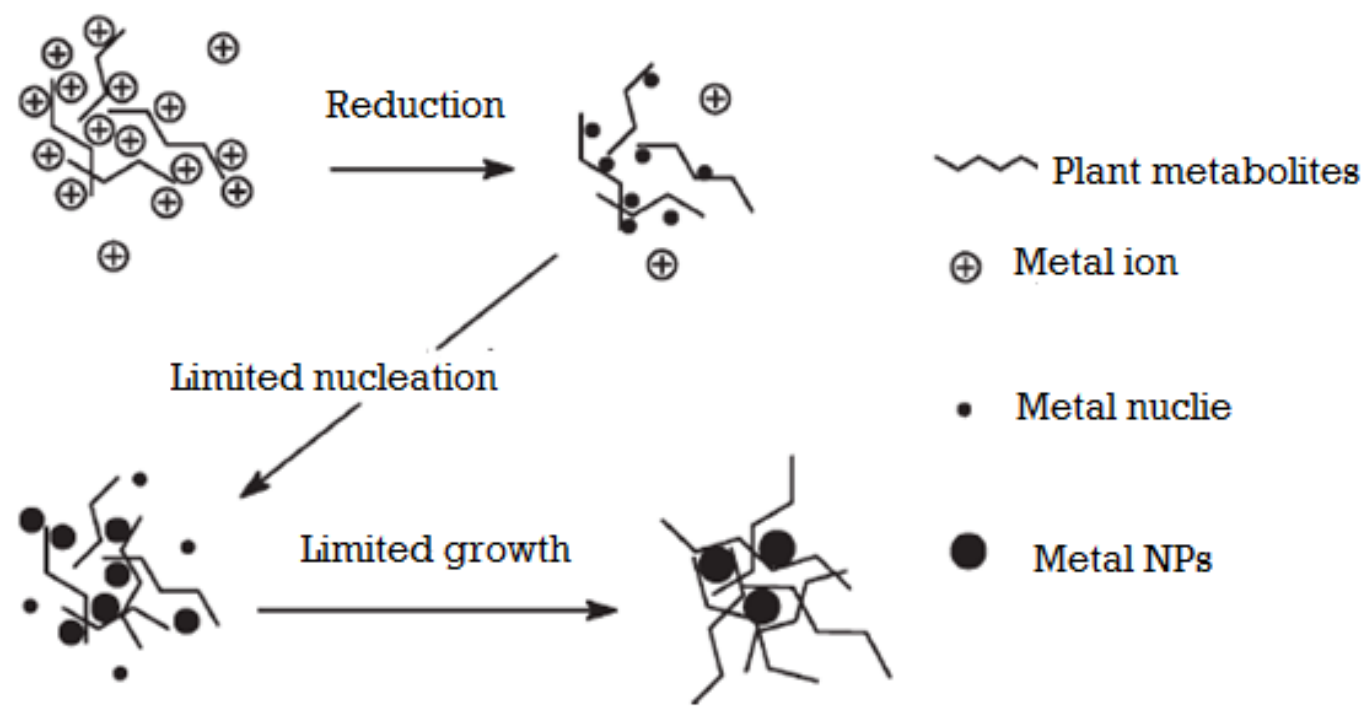

Fig 5:- Schematic representation of the formation process of metal NPs in the reaction solutions containing plant phytochemicals [12].

The major class of phytochemicals that is responsible for the bioreduction of metal ions is the polyols. Polyols are derivatives of phenolic compounds that contain multiple hydroxyl groups. One of the polyols compounds that are present in plants is the terpenoid family, including geraniol and tannic acid. The main function of phenolic compounds and polyols in plant cells is to act as antioxidants. They work together to absorb and neutralize free radicals, such as reactive oxygen species (ROS), thus, preventing oxidative damage of cellular components, aging and further cell death. The ability of polyols to scavenger free radicals is attributed to their redox capacity. The hydroxyl group of these compounds (phenolic form) can be oxidized to carbonyl group (quinone form) and hence, acts as a reducing agent (Fig 6.a). Such redox capacity is responsible for the bioreduction of gold and silver ions [10, $12,13]$. Shafaepourt et al. were able to extract geraniol from plant leaves. It was revealed that geraniol extract results in the bioreduction of silver ions, forming silver NPs in the size range of 1-100 nm (Fig 6.b) [12]. 
a-

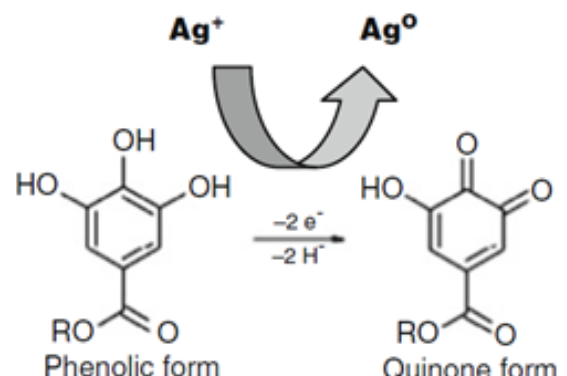

b-

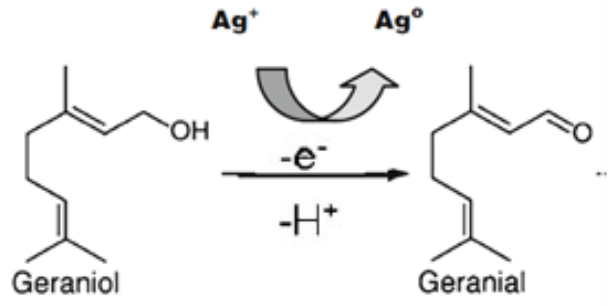

Fig 6:- Possible metabolites involved with the biosynthesis of silver nanoparticles in plants. a-Polyol reduction of silver ions, b- Gereniol as a reducing agent for silver ions [12].

Plant extract as a capping agent: The main reason why plants extract work so well in the synthesis of nanoparticles is that they act as a reducing agent during NPs synthesis as well as the postsynthesis stabilizing ligands. So, there is no need to use chemical surfactants as capping agents. Such nanoparticles stabilized by biological capping agents could further be utilized for biomedical applications $[2,9,14]$.

A number of studies have been done to address the biomolecules in plant that act as a capping agent. Fourier transforms Infrared spectroscopy (FT-IR), energy dispersive X-ray chemical analysis, and high-performance liquid chromatography indicated that carbohydrates in the plant extract are the primary nanoparticle stabilizing moieties [2]. In a study to determine the active species in garlic extract responsible for stabilizing silver ion ATR-FTIR was performed. ATR-FTIR spectra showed large $-\mathrm{OH}$ and $-\mathrm{CH}$ stretches obtained for the dried garlic extract (3300 and $2930 \mathrm{~cm}-1)$ and for the garlic extract-prepared silver colloidal suspension $(3270$ and $2930 \mathrm{~cm}-1)$. These $-\mathrm{OH}$ stretches are characteristic of sugar present in garlic. The primary nonstructural sugars that are readily extracted from garlic are sucrose and fructose. It was suggested that these nonstructural sugars function as stabilizers in NPs synthesis. This hypothesis was further supported by HPLC characterization of garlic extract and digested NPs solution. In garlic extract, fructose and sucrose showed an overlapped retention peak, centered at $1.15 \mathrm{~min}$. On the other hand, a retention time of $1.22 \mathrm{~min}$ was measured for the digested NPs solution. This slightly higher retention time indicates that water soluble carbohydrates, present in garlic, were bound to the surface of the silver nanoparticles [14].

\section{Control of size, morphology shape and monodispersity of nanoparticles:-}

As known, particle sizes directly affect the properties of nanoparticles. Moreover, monodispersity is also an important characteristic of the nanoparticles. The control of size and monodispersity is a major challenge of NPs biosynthesis. Several studies have revealed that controlling reaction parameters, such as type of plant extract, concentration of metal ions (substrate) and temperature can result in the size and monodispersity control of nanoparticles. UV-visible absorption spectroscopy is one of the main techniques used for the characterization of NPs in aqueous suspensions. Criterion for selecting optimum conditions is observing for a sharp peak at the characteristic wavelength (540 nm for AuNps and $420 \mathrm{~nm}$ for AgNps), showing maximum absorbance. In addition other characterization techniques can be used including, transmission electron microscope (TEM). TEM provides information about the size and size distribution of synthesized NPs $[6,8,15]$. 


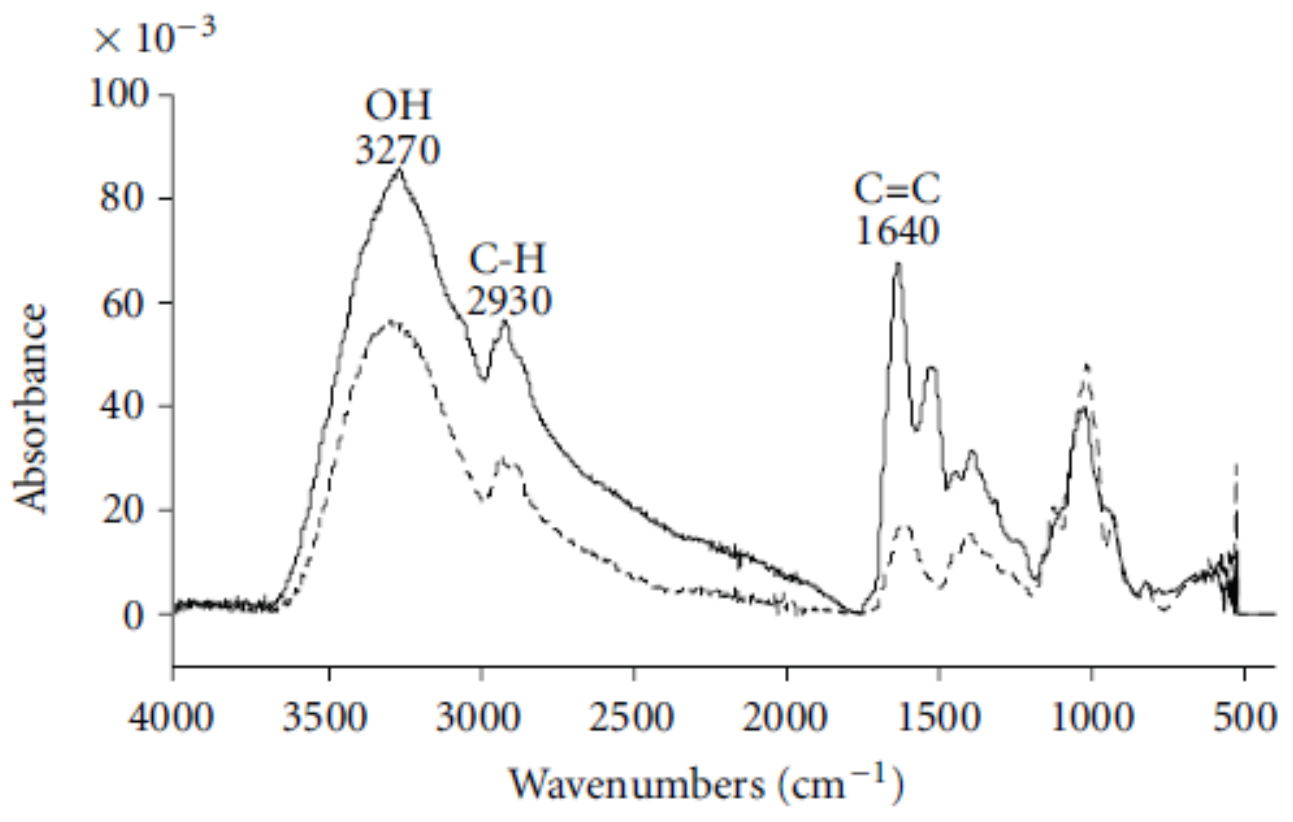

- AgNPs

- - Garlic extract

Fig 7:- Representative ATR-FTIR spectra from dried garlic extract and dried garlic extract prepared AgNPs [14].

\section{Effect of extract Concentration:-}

Generally speaking, the size and size distribution of metallic colloids varies significantly with the types of reduction reagents used. Strong reduction reagent promotes a fast reduction reaction. Consequently, large amount of metal atoms are generated in a short period of time i.e. rapid nucleation takes place. This would favor the formation of smaller NPs. On the other hand, weak reduction reagent induces a slow reaction rate and favors relatively larger particles [2, 6]. In the biosynthesis of metal nanoparticles, plant extract is the reduction reagent. By varying the type of plant extract, different effects are observed on the size and size distribution of metallic colloids, depending on the reduction potential of the phytochemical, acting as reducing agents. For example, Hydrocinnamic acid, caeffic acids are more efficient scavenger of free radicals (stronger reduction reagent) than benzoic derivatives present in plant extracts $[8,16]$.

It was revealed that large quantities of bark extract of Pinus eldarica lead to an increase in peak absorbance in UV/Vis spectrum. This indicated that by increasing the extract concentrations, nanoparticle production was also increased, but this relationship was not linear (Figure 8). Moreover, decrease in particle size of Ag NPs has been observed due to an increase in extract amount [15]. This results correlate with that obtained with Chenopodium album. With increasing leaf extract quantities in case of C. album; there was a decrease in particle size of the nanocolloids of silver and gold [16]. 


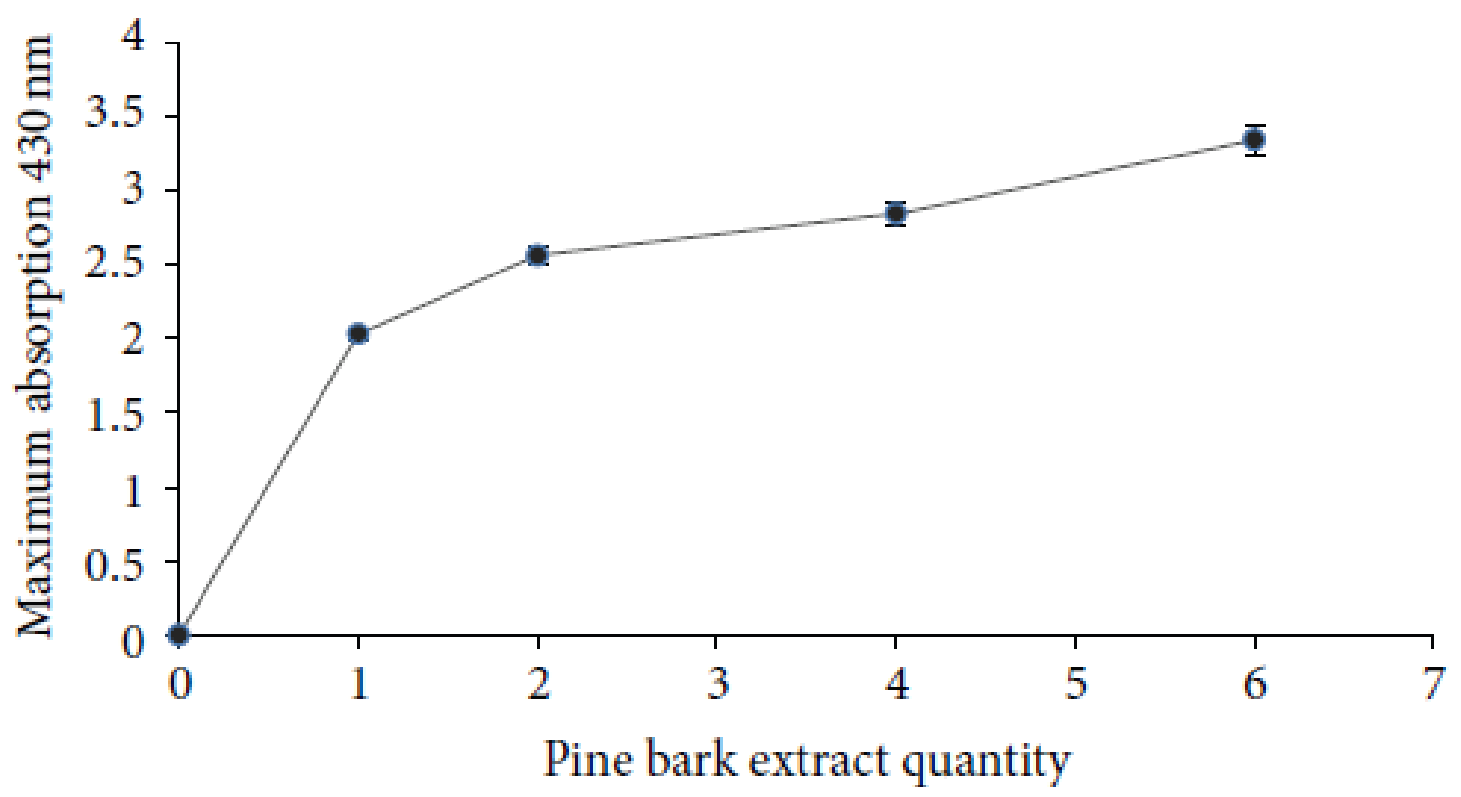

Fig 8:- Effect of different quantities of P. eldarica bark extract on the UV-VIS absorption spectra of AgNPs [15].

Contrary, lower garlic extract concentrations yield smaller and more monodisperse AgNPs . This was observed through a noticeable UV-VIS peak broadening (Fig 9). The peak broadening is attributed to an increase in polydispersity as a result of increasing garlic extract solution employed during synthesis. Furthermore, TEM analysis of the garlic extract-prepared AgNPs confirms a slight increase in nanoparticle size from $3.7 \pm 0.9 \mathrm{~nm}$ for $1.0 \mathrm{~mL}$ extract, $3.8 \pm 1.3 \mathrm{~nm}$ for $1.5 \mathrm{~mL}$ extract, and $4.1 \pm 1.5 \mathrm{~nm}$ for $2.0 \mathrm{~mL}$ extract (Fig 10). These studies provide evidence that size and size distribution of biosynthesized nanoparticles is a function of the type of extract used as well as the concentration of this extract [14].

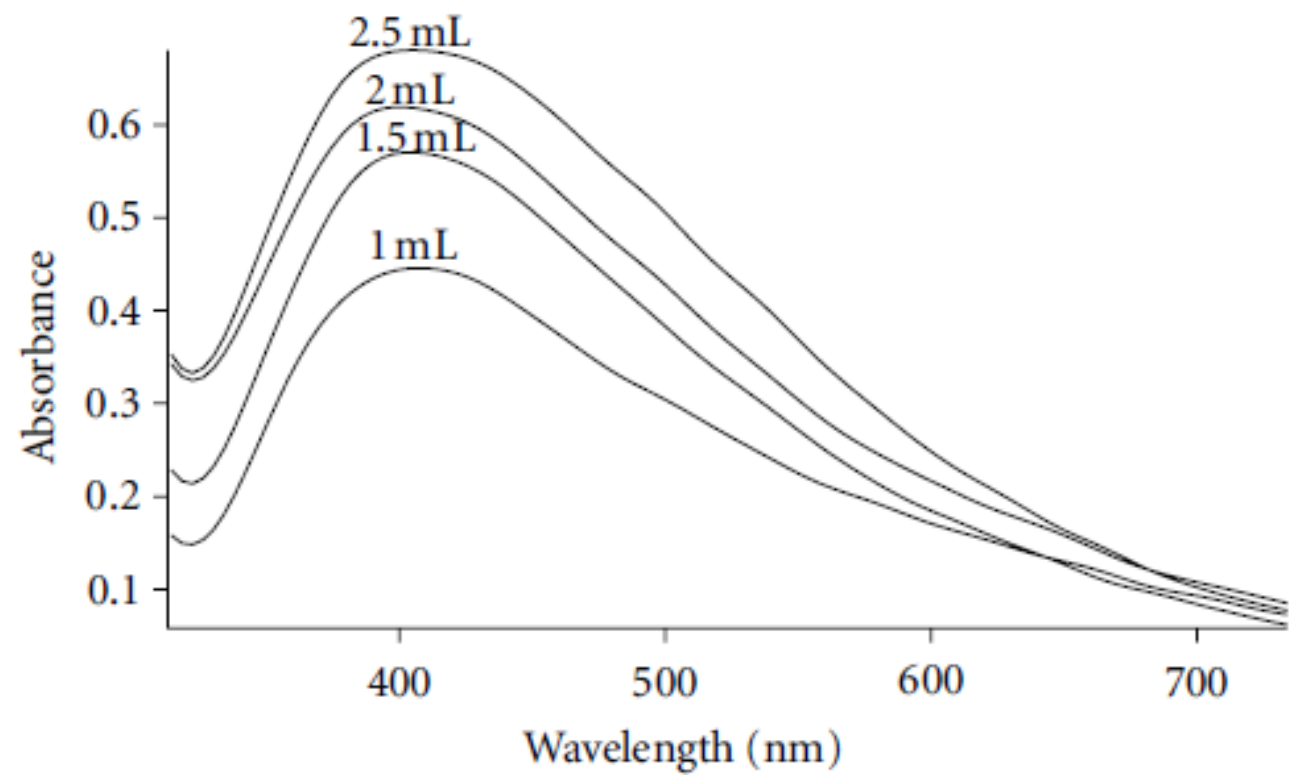

Fig 9:- UV-VIS absorbance spectra of silver nanoparticles prepared using (a) varying amounts garlic extract (1.0, $1.5,2.0$, and $2.5 \mathrm{~mL}$ ) [14]. 

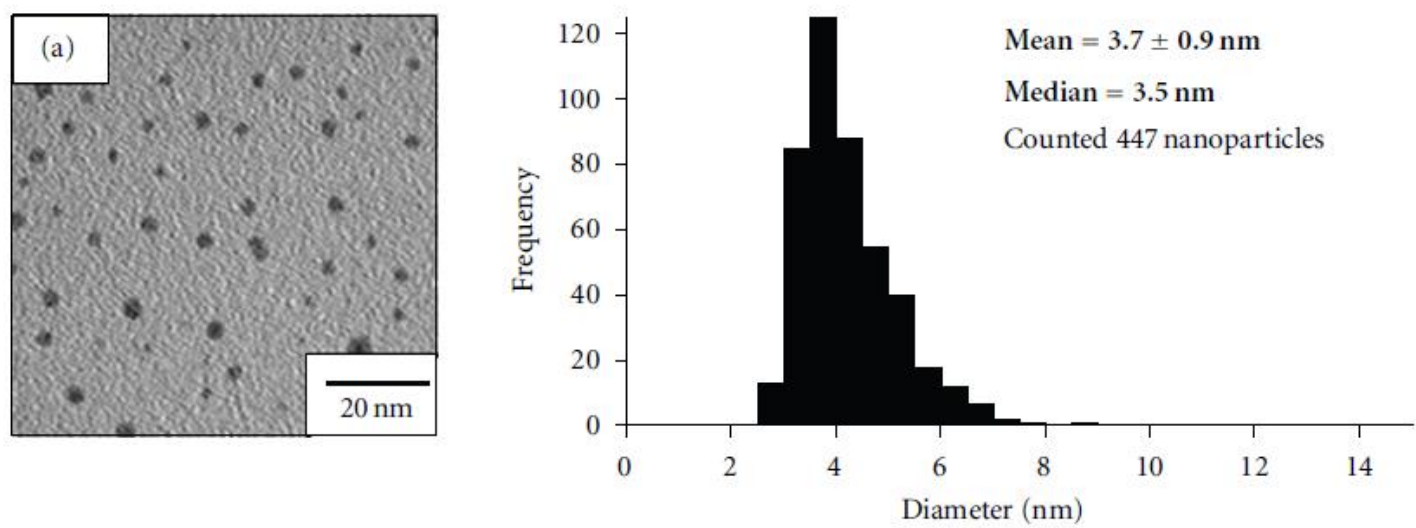

(a)
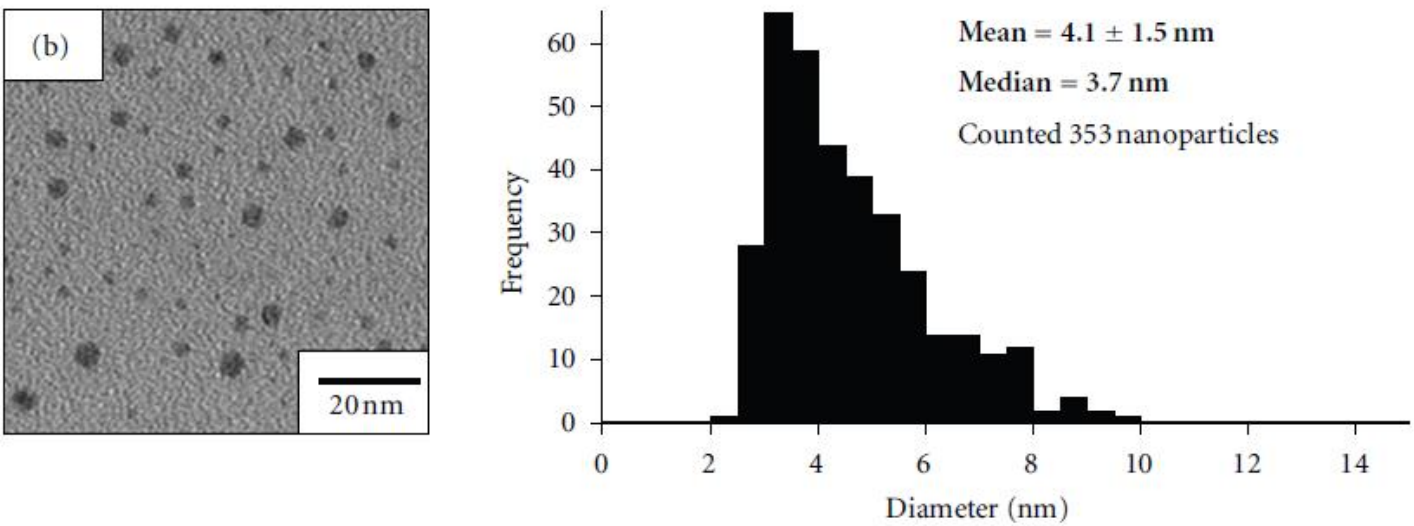

(b)

Fig 10:- Representative TEM images and histograms for AgNPs, synthesized using garlic extract (a) 1.0mL of garlic extract and (b) $2.0 \mathrm{~mL}$ of garlic extract [14].

It is not only the size and size distribution that can be controlled by varying concentration of plant extract, also the shape of biosynthesized nanoparticles can be changed with extract concentration. This was revealed in a study done on using phyllanthin extract for synthesizing AuNps and AgNps. Phyllanthin is a major bioactive lignan component of Phyllanthus amarus. The stabilizations of gold and silver NPs by phyllanthin have been kinetically monitored and characterized using Fourier transform infrared spectroscopy (FT-IR). Generally, the methoxy group

$\left(-\mathrm{OCH}_{3}\right)$ of phyllanthin shows a band at $1,088 \mathrm{~cm}^{-1}$. The FT-IR studies demonstrated a shift in the methoxy band in case of digested phyllanthin-stabilized AgNPs and AuNPs. This change can be attributed to the binding of the $\mathrm{OCH}_{3}$ group with the nanoparticles, due to the electron-donating nature of- $\mathrm{OCH}_{3}$ group of the phyllanthin. Moreover, the shape of phyllanthin extract-stabilized NPs vary by varying the concentration of phyllanthin extract. TEM analysis showed that at low concentrations of phyllanthin extract, AgNPs and AuNPs exhibit a hexagonal shape, while at higher concentrations, the shape changes to spherical particles (Fig 11) [17]. This can be attributed to the availability of phyllanthin stabilizer. At low concentrations, limited amount of Phyllanthin are adsorbed on surfaces of NPs having higher surface energies, thus limit particle growth along these surfaces. In other words, the NPs preferentially grow along certain surfaces, resulting in a hexagonal shape. At higher concentrations, enough amount of phyllanthin is available. So, they are adsorbed equally on the surface of NPs, thus, hinder particle growth along all surfaces to the same extent, resulting in spherical shape [2]. 


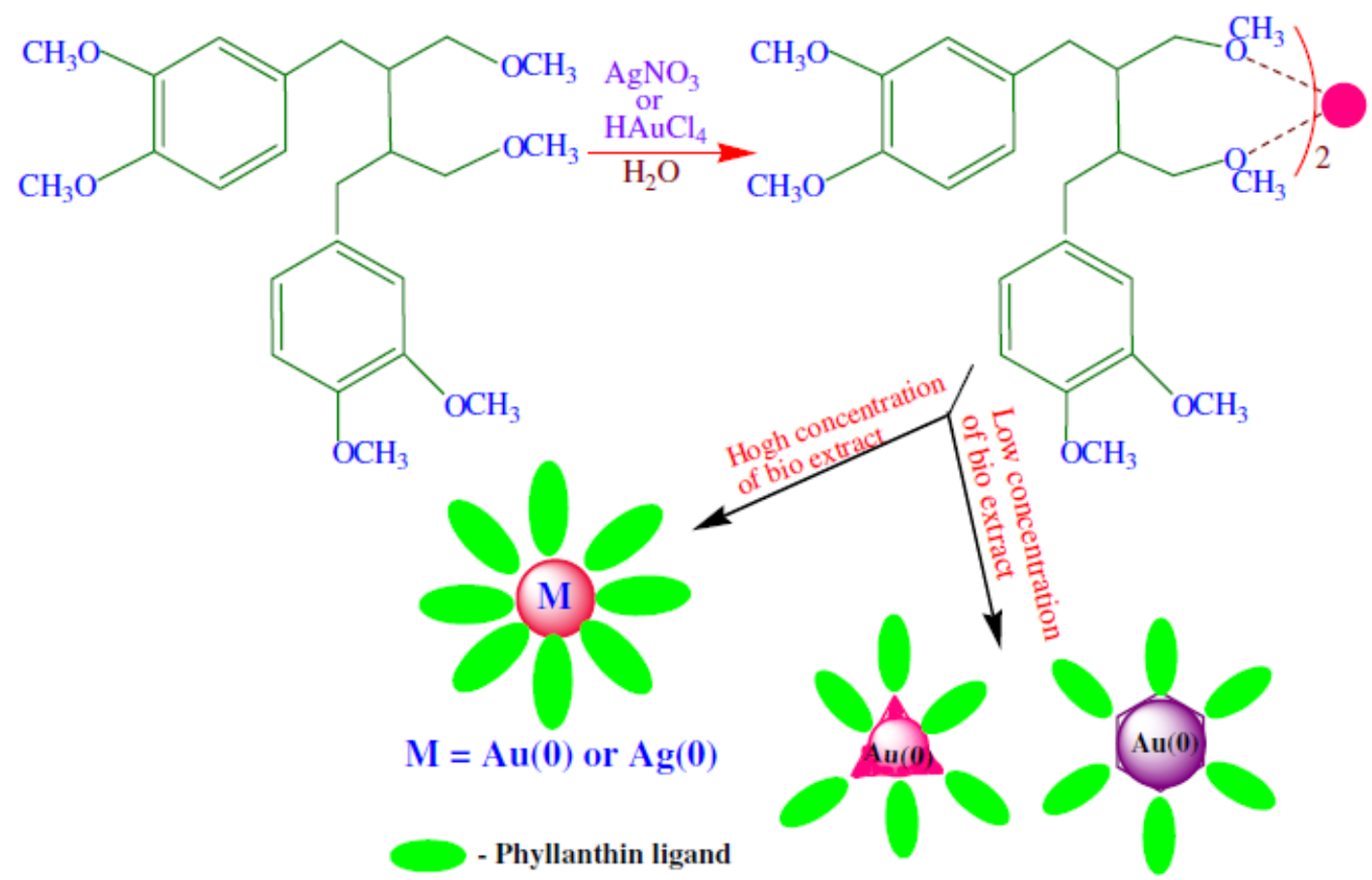

Fig 11:- Schematic diagram of the formation of phyllanthin stabilized gold and silver nanoparticles [17].

\section{Effect of substrate concentration:-}

As it was mentioned previously, the common approach used for the synthesis of metal NPs applies using of lower concentrations of precursor. At higher concentrations, the availability of growth species in the solution is high. In other words, there are enough amounts of growth species to be incorporated in the growing particles. So, if the colloidal particles are given the enough time in contact with its mother liquor, particles will tend to grow larger than in case of lower concentration (enough growing units for growth). Consequently, what originally started as small nuclei will dissolve with the resulting growth species precipitating on the surface of larger particles (Ostwald ripening). On the other hand, Lower concentrations results in diffusion-limiting growth, which intern promote the formation monosized NPs.

In addition, Rapid addition of solute successfully promotes nucleation over particle growth [2,5].This is also applied for the biosynthesis of metal nanoparticles using plant extract. Up on using the leaf extract of Chenopodium album, TEM analysis showed that with increasing the concentration of the metal ions, from 1:30 $(0.1-5 \mathrm{mM})$ ratio, results in an increase in particle size, as well as poly-diversity (Fig 12). At higher concentrations (5mM), large AgNPs are formed, varying from $40-90 \mathrm{~nm}[8]$.

\section{Effect of Temperature:-}

It was reported in a number of studies that reaction temperature can impact nanoparticle size, polydispersity, and reaction kinetics as well. In order to investigate the effect of temperature, the reaction mixture, containing Pinus eldarica bark extract for the synthesis of silver nanoparticles, was heated at different temperatures $(25 \circ \mathrm{C}, 50 \circ \mathrm{C}$, $100 \circ \mathrm{C}$, and $150 \circ \mathrm{C})$. Samples were collected from the reaction mixture, and UV-VIS spectra were determined. By increasing reaction temperature, an increase in absorbance was observed, which reflects an increase in the production of colloidal silver nanoparticles (Fig. 13) [15].

Moreover, when the reaction temperature was increased from 25 to $150^{\circ} \mathrm{C}$, the size of AgNPs became smaller which resulted into sharpness of UV-VIS band [15]. This results correlate with that obtained in case of Chenopodium album leaf extract. Different absorbance spectra of AgNPs and AuNPs were taken at different temperature. The peak sharpness increases with an increase in the reaction temperature (Fig. 14.a and b). Most likely this occurs due to increase in reaction rate of the conversion of the metal ion to nanoparticles by the effect of high temperature [8]. 

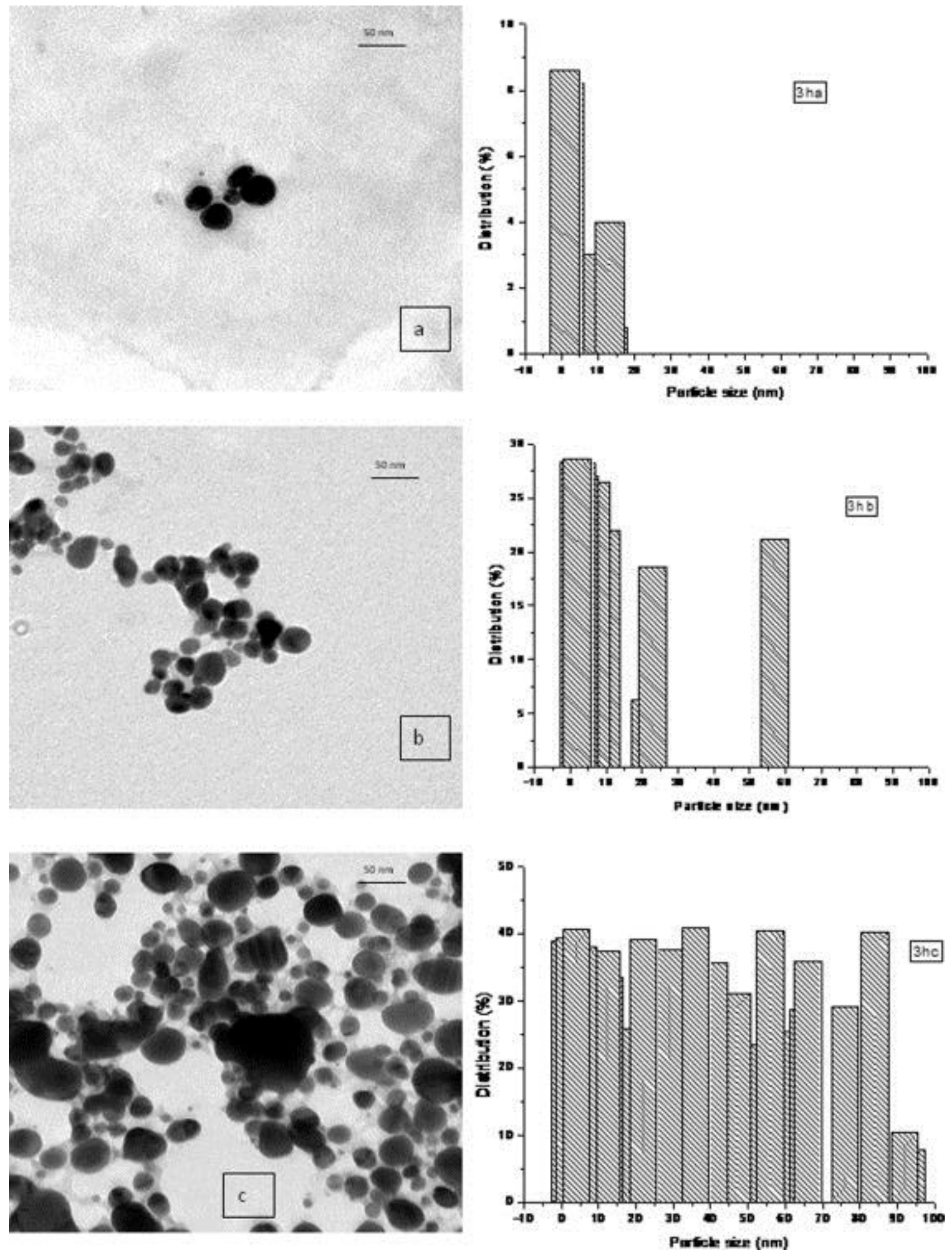

Fig 12:- TEM images of AgNPs at different silver ion solution $(0.1,1,5 \mathrm{mM}$; leaf extract/metal ion concentration ratio, 1:30; contact time $30 \mathrm{~min}$ ) and their histograms (a) at $0.1 \mathrm{mM}$, (b) at $1 \mathrm{mM}$ and (c) at $5 \mathrm{mM}$ [8]. 


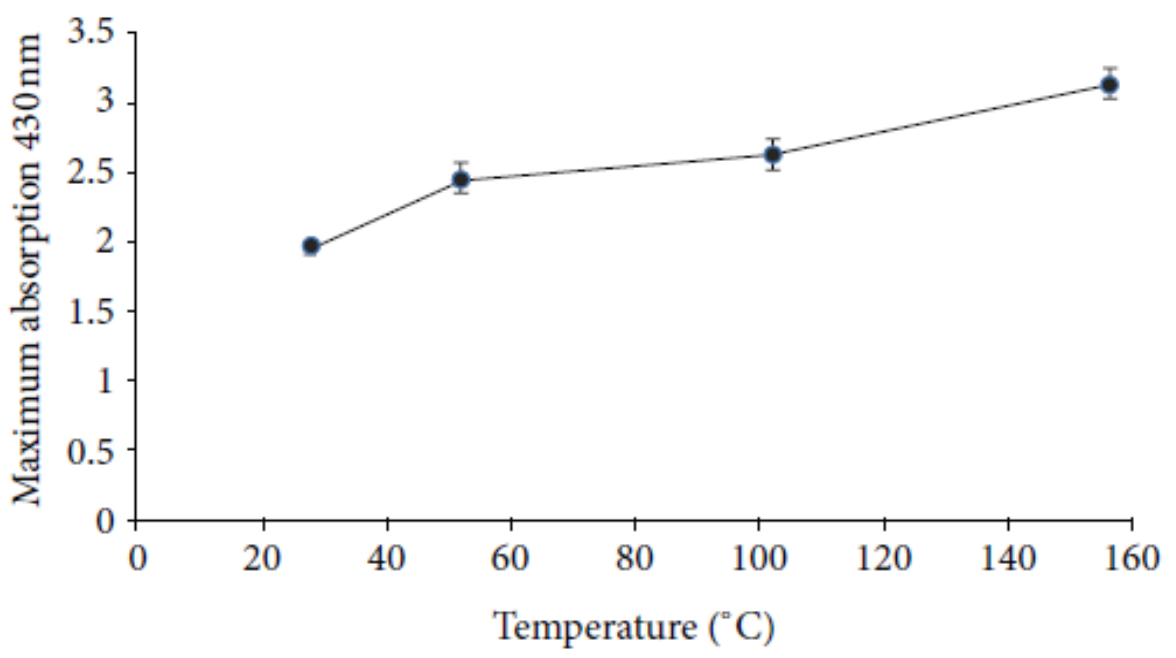

Fig 13:- Effect of reaction temperature on UV-VIS absorption spectra of AgNPs [15].
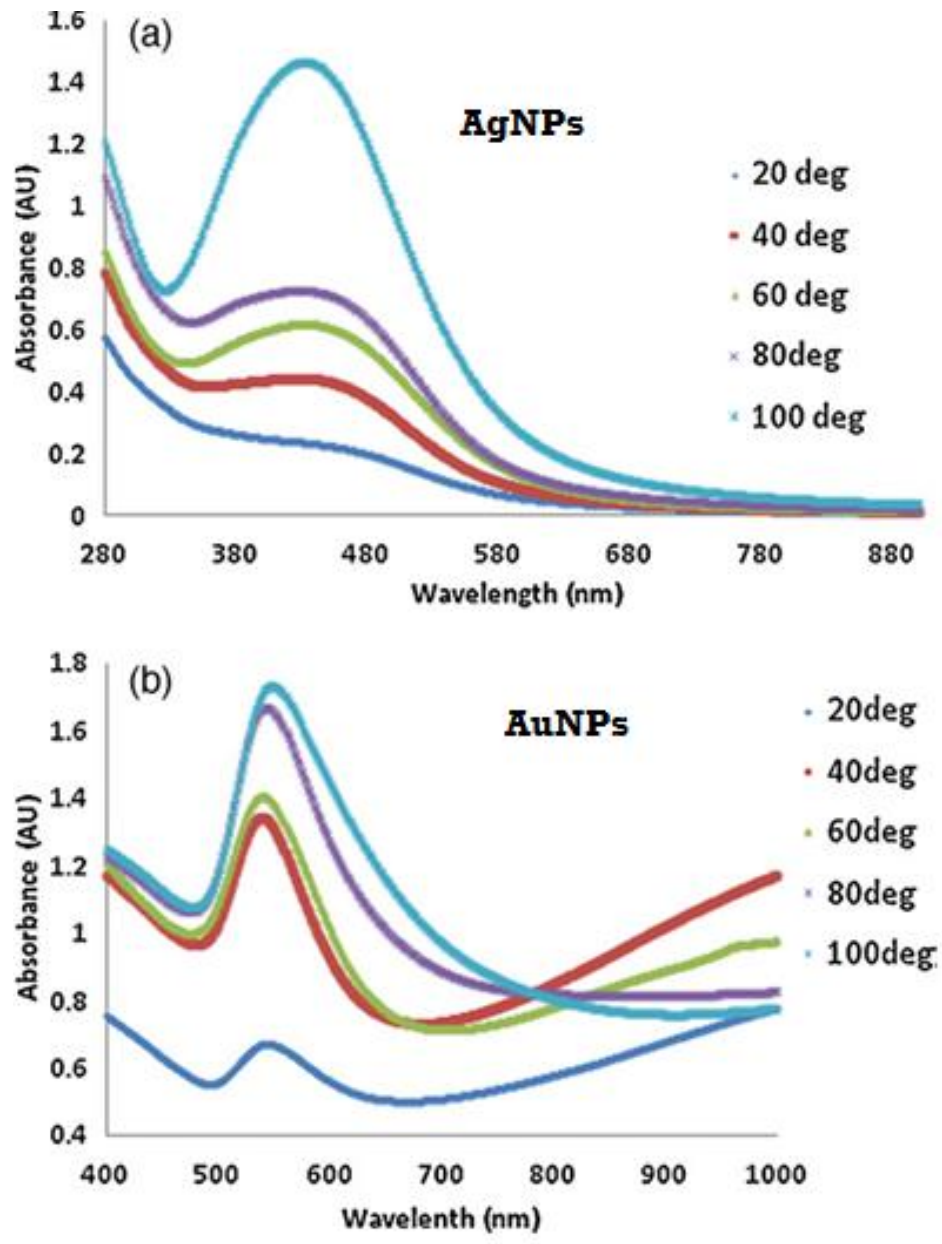

Fig 14:- Effect of reaction temperature on NPs synthesis (leaf extract/metal ion concentration ratio, 1:30; contact time 30min; temperature 20, 40, 60, 80, $100{ }^{\circ} \mathrm{C}$ ), (a) AgNPs and (b) AuNPs [8]. 


\section{Metal nanoparticles in drug Delivery:-}

Targeted drug delivery refers to the use of carrier molecules to deliver drug precisely and safely to their target sites, thus resulting in a maximum therapeutic effect. Drug delivery can efficiently reduce the dosage of anti-cancer drugs and provide better specificity and low toxicity. An efficient drug delivery system must fulfill the following criteria: 1) first, is the high stability in physiological media in order to prevent aggregation, 2) high biocompatibility, so that it can navigate through blood-tissue barriers and reach target cells, without being removed with blood circulation, 3) adequate drug loading capacity in order to deliver sufficient drug concentration in the target cells, 4) finally, to exhibit low toxicity $[1,18]$. In this view, gold and silver nanoparticles represent a suitable candidate for the development of an effective drug delivery system (DDS). Owing to their small size, nanoparticles drug carriers can pass through blood-tissue barriers and the tight epithelial junctions that normally impede the delivery of high molecular weight drugs to the desired target site. In addition, their high surface area to volume ratio provides it with high drug-loading capacity. This would improve the pharmacokinetics and biodistribution of drug and thus, minimize drug toxicity by preferentially accumulating drug at the target site $[3,14,19]$.

\section{Green approach vs. chemical Approach:-}

Chemically or physically prepared metal nanoparticles show number of disadvantages which limit their use in drug delivery. Contrary, these limitations are not an issue when it comes to biosynthesized nanoparticles.

Enhance stability in biological media: In general, biological media contains certain components, such as glucose, amino acids, salts, vitamins and so forth, similar to in vivo conditions. It has been demonstrated that biological media, necessary for cellular growth and proliferation, causes irreversible aggregation of chemically-prepared nanoparticles [20, 21]. For example, Greulich et al found that AgNPs, stabilized by polyvinyl pyrrolidine (PVP), aggregate once exposed to biological media [21]. A comparative study was done to investigate the stability of garlic extract stabilized AgNPs against citrate stabilized AgNPs. In this study, AgNPs synthesized with $1.5 \mathrm{ml}$ of garlic extract was added to DMEM biological cell culture media in a volume ratio 1:1. The stability of AgNPs dispersion was detected as a function of time using UV-VIS analysis. Citrate stabilized NPs were investigated for comparison (Fig. 15). UV-VIS absorbance spectra for the garlic extract AgNPs exhibit a decrease over $18 \mathrm{hr}$. This decrease in absorbance is due to reversible clustering, since gentle mixing of the suspension readily resuspends the NPs back to solution. After $18 \mathrm{hr}$ of incubation in DMEM biological media, the characteristic absorbance peak (404 nm) remain prominent (Fig. 15.a). Furthermore, visual inspection of the garlic stabilized AgNPs dispersion showed no signs of aggregation. On the other hand, citrate-stabilized nanoparticles showed a significant decrease in absorbance intensity in less than 30 minutes after the addition of DMEM. After $15 \mathrm{hr}$ of incubation, the AgNPs have completely and irreversibly precipitated out of the solution (Fig. 15.b) [14].

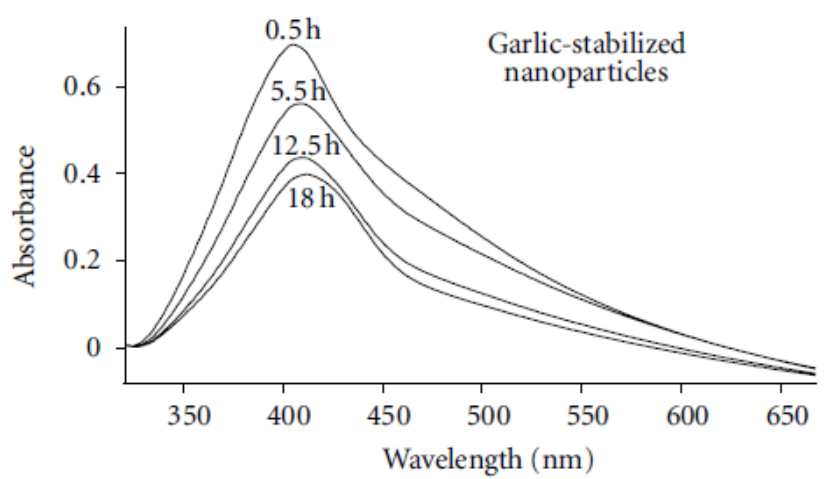

(a)

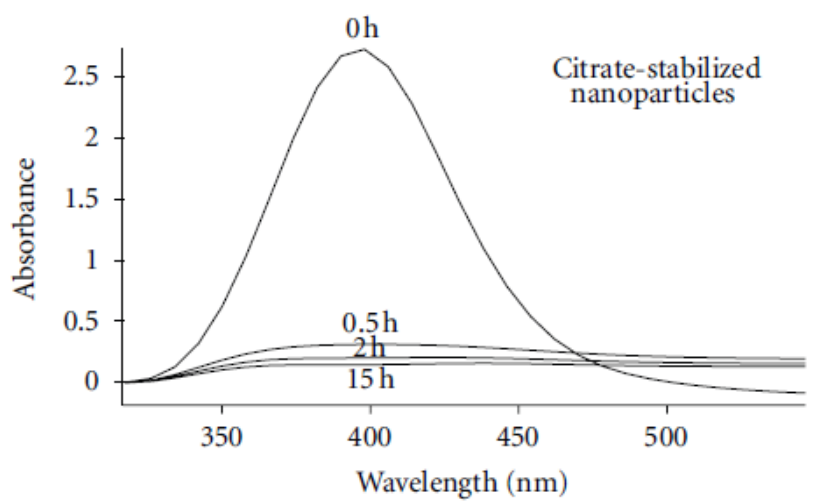

(b)

Fig 15:- UV-VIS spectra for silver nanoparticles stabilized by (a) garlic extract and (b) citrate in the presence of DMEM/high modified cell culture media as a function of time [14].

Cytotoxicity: Chemical synthesis methods lead to the presence of toxic chemicals adsorbed on the surface of NPs that have adverse effects in medical applications. For example, the stabilizers used in chemical synthesis, such as polyvinyl alcohol (PVA), polyethylene glycol (PEG) and polyvinyl pyrrolidine (PVP), remain on the surface of NPs 
even after several washings, which can cause toxicity. This is not the case in biosynthesis, where is there no need to use toxic chemicals since plant extract serves as both reducing agent and stabilizer [22].

Ease of synthesis: Chemically synthesized metal NPs requires several steps of surface modifications. The NPs are functionalized with different bio-moieties to render it biocompatible for medical purposes. On the other hand, employing natural reagents during biosynthesis improves biocompatibility of NPs. Consequently, there is no need for surface modifications or extensive post-synthesis purification as compared to conventional methods [23].

Resistance to reactive oxygen species: It has been demonstrated that living cells exposed to nanoparticles can produce reactive oxygen species (ROS), such as $\mathrm{OH}^{-}$and $\mathrm{H}_{2} \mathrm{O}_{2}$, as a stress response. Exposure to these ROS can result in the oxidization of nanoparticles followed by irreversible aggregation; thus, render the application of NPs useless. Figure 16 shows the UV-VIS spectra of citrate and garlic extract stabilized AgNPs in presence of $\mathrm{H}_{2} \mathrm{O}_{2}$. Upon exposure to $\mathrm{H}_{2} \mathrm{O}_{2}$, citrate-stabilized AgNPs suspension turns clear immediately. The loss of SRP absorbance peak indicates for the oxidation of $\mathrm{Ag}^{0}$ to $\mathrm{Ag}^{+}$. This in turn, reflects the poor oxidation resistance of citrate-stabilized NPs (Fig.16.a). Contrary, the SRP absorbance peak of garlic-stabilized AgNPs is still present after addition of $\mathrm{H}_{2} \mathrm{O}_{2}$ (Fig.16.b) [14].The oxidation resistance of garlic extract AgNPs may be attributed to the presence of phytochemicals in the plant extract, having strong antioxidant activity, such as allicin. Such antioxidant compounds can scavenger reactive oxygen species $[9,14]$.

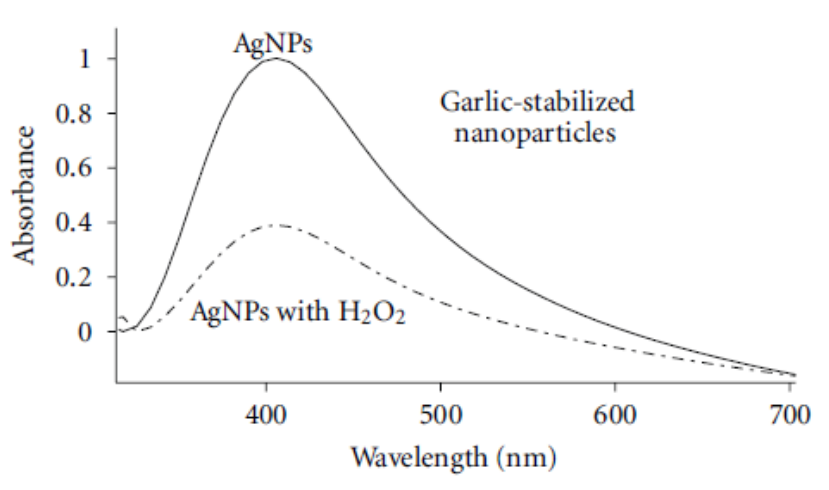

(a)

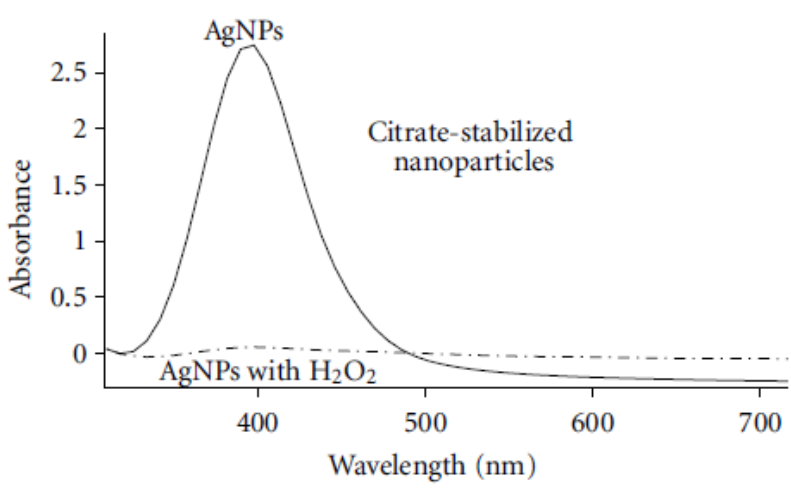

(b)

Fig 16:- UV-VIS spectra for silver nanoparticles stabilized by (a) garlic extract, (b) citrate [14].

\section{Current research and future prospects:-}

Green synthesis of metal NPs is considered a new approach. There are a number of researches that evaluate the potential of AgNPs and AuNPs as a drug delivery system (DDS), especially in cancer treatment. Mukherjee. et al established a single-step green chemistry approach for the synthesis of biocompatible gold nanoparticles (AuNPs) from chloroauric acid $\left(\mathrm{HAuCl}_{4}\right)$, using a water extract of Eclipta Alba leaves. The AuNPs using Eclipta extract have been formed in very short time, even in less than 10 min. The obtained AgNPs was further loaded with doxorubicin (DOX), a FDA approved anticancer drug, to design a DOX-loaded drug delivery system (Au-DOX). Administration of this DDS to breast cancer cells (MCF-7 and MDA-MB-231) shows significant inhibition of breast cancer cell proliferation compared to naked doxorubicin [24]. Moreover, the use of nanoparticles in drug delivery therapy holds much promise in targeting remote tissues, including brain tissue affected by Alzheimer disease. One of the biggest problems and challenges in the development of new drugs for treatment of Alzheimer is the difficulty of passing the drugs across the blood brain barrier (BBB). Aliev et al. established a study to test the potential of silver nanoparticles to leak on the $\mathrm{BBB}$, and therefore investigate the possibility of penetrating into the areas of the brain which are most damaged by Alzheimer disease. The study indicated that silver nanoparticles injected through the brain of test animals (intra-peritoneal area of brain) are definitely able to cross the BBB and can penetrate into the cell cytoplasm. Furthermore, it can induce underlying cellular changes, which can be used for drug delivery [25]. Therefore, it is strongly believed that the use of biosynthesized metal nanoparticles can offer large-scale production of biocompatible AuNPs that can be used as a delivery vehicle for different diseases. 


\section{Conclusion:-}

The common protocols used for the synthesis of metal nanoparticles often require the use of harsh chemicals, which result in the formation of toxic by-products during synthesis. The biosynthesis of metal nanoparticles using plant extract eliminates the use of hazardous reagents. In other words, it affords greener, safer and cost effective alternative. This eco-friendly approach is considered an optimal candidate for biomedical research applications in drug delivery. More researches are focusing on the different parameters that can alter size, shape and monodiversity of nanoparticles, in order to reach optimal conditions for biosynthesis.

\section{References:-}

1. Sadowski, Z., (2010). Biosynthesis and Application of Silver and Gold Nanoparticles. Silver Nanoparticles. InTech. 258-276.

2. Cao, G., \& Ebrary, I. (2004). Zero-Dimensional Nanostructures: Nanoparticles. Nanostructures \& nanomaterials: Synthesis, properties \& applications. London: Imperial College Press. 61-141.

3. Schröfel, A., \& Kratošová, G., (2011). Biosynthesis of Metallic Nanoparticles and Their Applications. Intracellular delivery: Fundamentals and applications. Dordrecht: Springer Netherlands. 373-409.

4. Arunachalam, D.K., \& Annamalai, K., S., (2013). Chrysopogon zizanioides aqueous extract mediated synthesis, characterization of crystalline silver and gold nanoparticles for biomedical applications. International Journal of Nanomedicine, 8: 2375-2384.

5. Zhang, J.Z. (2009). Synthesis and Fabrication of Nanomaterials. Optical properties and spectroscopy of nanomaterials. Hackensack, NJ: World Scientific. 77-115.

6. Hei, H., Zhang, G., et al.(2012). Controlled Synthesis and Characterization of Noble Metal Nanoparticles. Soft Nanoscience Letters, 2: 34-40.

7. Guzmán, G. M., Dille, J. et al. (2009).Synthesis of silver nanoparticles by chemical reduction method and their antibacterial activity. International Journal of Chemical and Biological Engineering, 2: 104-11.

8. Dwivedi, A. D., \& Gopal, K. (2010). Biosynthesis of silver and gold nanoparticles using chenopodium album leaf extract. Colloids and Surfaces A: Physicochemical and Engineering Aspects, 369: 27-33.

9. Hebbalalu, D., Lalley, J., et al. (2013). Greener Techniques for the Synthesis of Silver Nanoparticles Using Plant Extracts, Enzymes, Bacteria, Biodegradable Polymers, and Microwaves. ACS Sustainable Chem. Eng, 1: 703-712.

10. Duran, N., \& Marcato, D.P. (2012). Biotechnological Routes to Metallic Nanoparticles Production. NanoAntimicrobials: Progress and Prospects. Heidelberg: Springer Berlin. 337-374.

11. Li, S., Shen, Y., Xie, A., et al. (2007). Green synthesis of silver nanoparticles using Capsicum annuиm L. extract. Green Chem, 9: 852-858.

12. Durán, N., Marcato, D.P., et al. (2011). Mechanistic aspects in the biogenic synthesis of extracellular metal nanoparticles by peptides, bacteria, fungi, and plants. Appl Microbiol Biotechnol, 90:1609-1624.

13. Ahmed, N., \& Sharma, S. (2012). Green Synthesis of Silver Nanoparticles Using extracts of Ananas comosus. Green and Sustainable Chemistry, 2: 141-147.

14. White, V.G.,Kerscher,P., et al. (2012). Green Synthesis of Robust, Biocompatible Silver Nanoparticles Using Garlic Extract. Journal of Nanomaterials, 2012: 1-12.

15. Iravani S., \& Zolfaghari B. (2013). Green synthesis of silver nanoparticles using Pinus eldarica bark extract. BioMed Research International, 2013:1-5.

16. Sant, G., D., Gujarathi, R.T., et al. (2013). Adiantum philippense L. Frond Assisted Rapid Green Synthesis of Gold and Silver Nanoparticles. Journal of Nanoparticles, 2013: 1-9.

17. Kasthuri, J., Kathiravan, K., Rajendiran, N. (2009). Phyllanthin-assisted biosynthesis of silver and gold nanoparticles: a novel biological approach. JNanopart Res, 11:1075-1085.

18. Voliani, V., Signore, G., et al. (2012). Smart Delivery and Controlled Drug Release with Gold Nanoparticles: New Frontiers in Nanomedicine. Recent Patents on Nanomedicine, 2: 1-11.

19. Raghunandan, D., \& Ravishankar, B. (2011) Anti-cancer studies of noble metal Nanoparticles synthesized using different plant extracts. Cancer Nano, 2: 57-65. Pandey, S., Sharon, M., et al. (2012). Biosynthesis of Highly Stable Gold Nanoparticles Using Citrus limone. Annals of Biological Research, 3:2378-2382.

20. Pandey, S., Oza, G., et al. (2012). Green Synthesis of Highly Stable Gold Nanoparticles using Momordica charantia as Nano fabricator. Archives of Applied Science Research, 4: 1135-1141.

21. Kumari, A., Yadav, K.S., et al. (2012). Plant extract synthesized PLA Nanoparticles for controlled and sustained release of Quercetin: A Green approach. PLoS ONE, 7:1-10. 
22. Kumar, K.P., Paul, W., et al. (2011). Green synthesis of gold nanoparticles with Zingiber officinale extract: Characterization and blood compatibility. Process Biochemistry, 46: 2007-2013.

23. Mukherjee, S., Patra, S., et al. (2012). Green chemistry approach for the synthesis and stabilization of biocompatible gold nanoparticles and their potential applications in cancer therapy. Nanotechnology, 23: 455103.

24. Aliev, G., Daza, J., et al. (2009). Silver nanoparticles as alternate strategies for drug delivery to the Alzheimer brain. Alzheimer's \& Dementia, 5: P65. 